\title{
SIRT1 suppresses breast cancer growth through downregulation of the Bcl-2 protein
}

\author{
SHOU-JEN KUO ${ }^{1,2^{*}}$, HUI-YI LIN ${ }^{3 *}$, SU-YU CHIEN $^{4}$ and DAR-REN CHEN ${ }^{1,2}$ \\ ${ }^{1}$ Comprehensive Breast Cancer Center, Changhua Christian Hospital, Changhua 50006; ${ }^{2}$ School of Medicine, Chung Shan \\ Medical University, Taichung 40201; ${ }^{3}$ Department of Pharmacology, School of Pharmacology, China Medical University, \\ Taichung 40402; ${ }^{4}$ Department of Pharmacology, Changhua Christian Hospital, Changhua 50006, Taiwan, R.O.C.
}

Received December 1, 2012; Accepted March 6, 2013

DOI: $10.3892 /$ or.2013.2470

\begin{abstract}
Silent mating-type information regulation 2 homologue 1 (SIRT1), a member of the class III histone deacetylase (HDAC) family, is the mammalian ortholog of yeast Sir2. It has been reported to play a key role in a variety of physiological processes such as genomic stability, metabolism, neurogenesis and cell survival. The deacetylase function of SIRT1 has been suggested to play a role in prolonging the life of mammals. However, the suggested functions of SIRT1 as a potential tumor promoter have been challenged by observations of their respective downregulation and upregulation in various types of cancer. Breast cancer patients were included in the present study between 2007 and 2008. Their tumor tissues and paired normal breast tissues were collected and used for evaluation of the expression levels of SIRT1 and Ki67. The effects of SIRT1 on human breast cancer cell lines were also investigated. Immunohistochemistry showed that there is a high correlation between SIRT1 and Ki67 expression. Following treatment with sirtinol (inhibitor of SIRT1), the expression of the prosurvival protein Bcl-2 was markedly decreased in both MCF-7 and MDA-MB-231 cell lines, particularly in MDA-MB-231. Results of the present study revealed that inhibition of SIRT1 activity may be a promising chemotherapeutic strategy against breast cancer.
\end{abstract}

\section{Introduction}

Silent mating-type information regulation 2 homologue 1 (SIRT1) is a protomember of the sirtuin family (SIRT1-7) that is involved in a variety of physiological processes such as genomic stability, metabolism, neurogenesis and cell survival due to its ability to deacetylate both histone and numerous

Correspondence to: Professor Dar-Ren Chen, Comprehensive Breast Cancer Center, Changhua Christian Hospital, 135 Nanhsiao Street, Changhua 50006, Taiwan, R.O.C.

E-mail: darren_chen@cch.org.tw

*Contributed equally

Key words: Bcl-2, breast cancer, Ki67, silent mating-type information regulation 2 homologue 1 non-histone substrates such as gene silencing, metabolism, neuroprotection, and genomic stability $(1,2)$. SIRT1 acts as a deacetylase not only on histone but also by deacetylating cell cycle regulators such as p53 (3), p73 (4) and $\mathrm{Rb}(5)$. With regard to the growth of cancer cells, it has been reported that SIRT1 regulates cell proliferation, survival, and death, and plays a pivotal role in tumorigenesis and longevity.

SIRT1 prompts cell proliferation and plays an important role in the drug resistance of tumor cells, especially during chemotherapy. In humans, several types of cancer, including prostate $(6,7)$, breast cancer $(8,9)$, colon $(10,11)$, glioblastoma (12), lymphoma (13) and acute myeloid leukemia (14) have been demonstrated to have a significantly increased expression of SIRT1. Furthermore, a recent study showed that SIRT1 regulates the DNA damage signaling pathway through deacetylation of NBS1 to promote cellular survival (15). It was reported that SIRT1 upregulates the expression of multidrug resistance 1 (MDR1) in cancer, and this may cause cancer resistance to chemotherapy (16). SIRT1-deficient cells exhibited p53 hyperacetylation following DNA damage and increased ionizing radiation-induced thymocyte apoptosis in vivo along with downregulation of MDR1 (16-19). This evidence indicates the oncogenic role of SIRT1.

By contrast, several studies have shown the tumor suppressor role of SIRT1. The reduced transformation capability of c-myc can be compromised in the presence of SIRT1 (20). Ectopic induction of SIRT1 in a $\beta$-catenin-driven mouse model of colon cancer significantly reduced colon cancer formation, growth, and animal morbidity (21). Moreover, the mouse embryonic fibroblasts (MEFs) derived from SIRT1 ${ }^{-/}$mice are prone to spontaneous immortalization, suggesting that SIRT1 behaves as a growth-suppressive gene (22). Analysis of SIRT1 mutant mice revealed that SIRT1 ${ }^{-/}$MEFs displayed chromosome aneuploidy and repair defects, and that SIRT1 played an important role in the maintenance of the heterochromatin structure through deacetylation of histones in vivo (23). Collectively, these previous findings indicated that SIRT1 may possess both pro- and anti-proliferative functions, depending on the cell types. In spite of the controversial role of SIRT1 in tumorigenesis $(24,25)$, it is evident that SIRT1 is significantly involved in the process of tumorigenesis.

Since SIRT1 is involved in both the death and survival of cells, we proposed that SIRT1 may be a critical determinant 
of cell fate during tumorigenesis. To test this hypothesis, we investigated the expression of SIRT1 in tumor breast tissues and matched normal breast tissues of Taiwanese women, and used breast cancer cell lines to examine the role of SIRT1 in breast tumorigenesis.

\section{Materials and methods}

Patients. A total of 27 breast cancer patients were included in this study between 2007 and 2008. Tumor tissues and the paired normal breast tissues were immediately frozen after collection. The cases were diagnosed as infiltrating ductal carcinoma (IDC; $n=27$ ) according to the World Health Organization classification system (26). Clinical data were collected retrospectively. All patients provided informed consent and the study was approved by the Institutional Review Board of Changhua Christian Hospital.

Antibodies and reagents. The antibodies used for western blotting were: anti-SIRT1 (ab53517; Abcam, Cambridge, UK), anti- $\beta$-actin (A-5441) and anti-Bcl-2 (B-3170) (both from Sigma-Aldrich Co., MI, USA). The primary antibodies used for immunohistochemical analysis were: anti-SIRT1 (ab7343; Abcam) and anti-Ki67 (RM-9106-S1; Lab Vision, Fremont, CA, USA). Chemicals and reagents were purchased from Sigma-Aldrich Co., unless otherwise indicated.

Immunohistochemical analysis. The 5- $\mu \mathrm{m}$ sections of formalin-fixed, paraffin-embedded tissues were deparaffinized, rehydrated and the antigen retrieved. The tissue sections were incubated with hydrogen peroxide for $5 \mathrm{~min}$ at room temperature to quench the endogenous peroxidase. After blocking in normal goat serum, the tissue sections were incubated for $1 \mathrm{~h}$ at room temperature with the primary antibodies anti-SIRT1 (1:100 dilution) and anti-Ki67 (1:50 dilution), respectively. The slides were then washed with $0.5 \%$ Triton X-100 in PBS and incubated with HRP-conjugated secondary antibody for $1 \mathrm{~h}$ (Vector Laboratories, Burlingame CA, USA). Nuclei were counterstained with hematoxylin. As a negative control, normal goat serum was substituted for the primary antibody. All slides were examined by two independent investigators who were blind to the clinical data. To evaluate the expression of SIRT1 and Ki67 in tumor breast tissue and matched normal breast tissue, the staining intensity was scored from 0 to 3 . The ratio of the positively stained area to the total section area was assessed.

Reverse transcription-polymerase chain reaction (RT-PCR). Total RNA was isolated from breast tissue using TRIzol Reagent and cDNA was synthesized by MMLV Reverse transcriptase (both from Invitrogen, Carlsbad, CA, USA). The primer sequences used for PCR are shown in Table I. Synthesized cDNA was amplified and the PCR product was then visualized on $1 \%$ agarose gel. The intensity of the PCR product was determined by densitometry using the Gel Image Analysis System (Alpha Innotech Co., San Leandro, CA, USA).

Cell culture. The human breast cancer cell lines and MCF-7 and MDA-MB-231 cell lines were grown in Dulbecco's modified Eagle's medium: Nutrient Mixture F-12 (DMEM/F12)
Table I. Oligonucleotide sequences for RT-PCR.

\begin{tabular}{ll}
\hline Primer & \multicolumn{1}{c}{ Primer sequence } \\
\hline SIRT1 & F: 5'-CATCTCTCTGTCACAAATTCATAGCC-3' \\
& R: 5'-GTAATTTCTGAAAGCTTTACAGGGTT-3' \\
GAPDH & F: 5'-GAGTCAACGGATTTGGTCGT-3' \\
& R: 5'-GGTGCTAAGCAGTTGGTGGT-3'
\end{tabular}

SIRT1, silent mating-type information regulation 2 homologue 1; $\mathrm{F}$, forward; R, reverse.

supplemented with $10 \%$ fetal bovine serum, $2 \mathrm{mM}$ glutamine, $100 \mathrm{U} / \mathrm{ml}$ penicillin and $100 \mu \mathrm{g} / \mathrm{ml}$ streptomycin (Gibco, Invitrogen Ltd., Carlsbad, CA, USA) in a humidified atmosphere containing $5 \% \mathrm{CO}_{2}$ at $37^{\circ} \mathrm{C}$.

Cell proliferation assay. The cytotoxicity effect of sirtinol on MCF-7 and MDA-MB-231 cells was determined by trypan blue dye exclusion and Alamar-blue assays (AbD Serotec, Kidlington, UK). Briefly, the cells were plated at a density of $2 \times 10^{5}$ cells in 6 -well plates with an increasing dose of sirtinol (10-50 $\mu \mathrm{M}$ in DMSO) or vehicle alone. Following incubation for 24 or $48 \mathrm{~h}$, cells were collected and an aliquot of cell suspension was mixed with an equal volume of trypan blue, and cells were then counted under the microscope. The cell viability assay was performed following the instructions of the Alamar blue assay kit. In brief, cells were plated at a density of $2 \times 10^{3}$ cells in $200 \mu \mathrm{l}$ of complete medium in a 96-well plate and treated with increasing doses of sirtinol or vehicle alone. Each treatment was performed in triplicate. The cells were incubated for 24 or $48 \mathrm{~h}$ at $37^{\circ} \mathrm{C}$ in $5 \% \mathrm{CO}_{2}$. Five hours before the end of the culture, the Alamar blue reagent ( $20 \mu \mathrm{l}$ in phenol red free RPMI-1640 medium) was added into each well and incubated up to 24 or $48 \mathrm{~h}$. Absorbance of $590 \mathrm{~nm}$ wavelength emissions was recorded.

Flow cytometry analysis. Cell cycle analysis was performed with flow cytometry. Cells were grown at a density of $5 \times 10^{5}$ cells in 6 -well culture plates, treated with different concentrations of sirtinol for 24 and $48 \mathrm{~h}$, and maintained at $37^{\circ} \mathrm{C}$ in a $5 \% \mathrm{CO}_{2}$ incubator. Following treatment, the cells were gently trypsinized, added to the culture medium, and pelleted by centrifugation. The cell pellet was resuspended in $1 \mathrm{ml}$ PBS, washed at $1,500 \times \mathrm{g}$ for $30 \mathrm{~min}$ at $4^{\circ} \mathrm{C}$, and fixed in ethanol $(75 \% \mathrm{v} / \mathrm{v})$. The cells were then washed with PBS and labeled with propidium iodide/RNase A solution. Labeled cells were analyzed by flow cytometry (Epics XL-MCL Flow Cytometry; Beckman Coulter, Inc., Fullerton, CA, USA) and the analyses were performed using WinMDI 2.9 software.

Western blot analysis. The cells were harvested at 24 and $48 \mathrm{~h}$ following sirtinol treatment, as described above, and washed with PBS, respectively. The cells were lysed with ice-cold RIPA lysis buffer (1X TBS, $1 \%$ Nonidet P-40, $0.5 \%$ sodium deoxycholate, $0.1 \%$ SDS, $0.004 \%$ sodium azide with protease inhibitor) and harvested. The lysate was centrifuged at $14,000 \mathrm{x} \mathrm{g}$ for $30 \mathrm{~min}$ at $4^{\circ} \mathrm{C}$, and the supernatant 


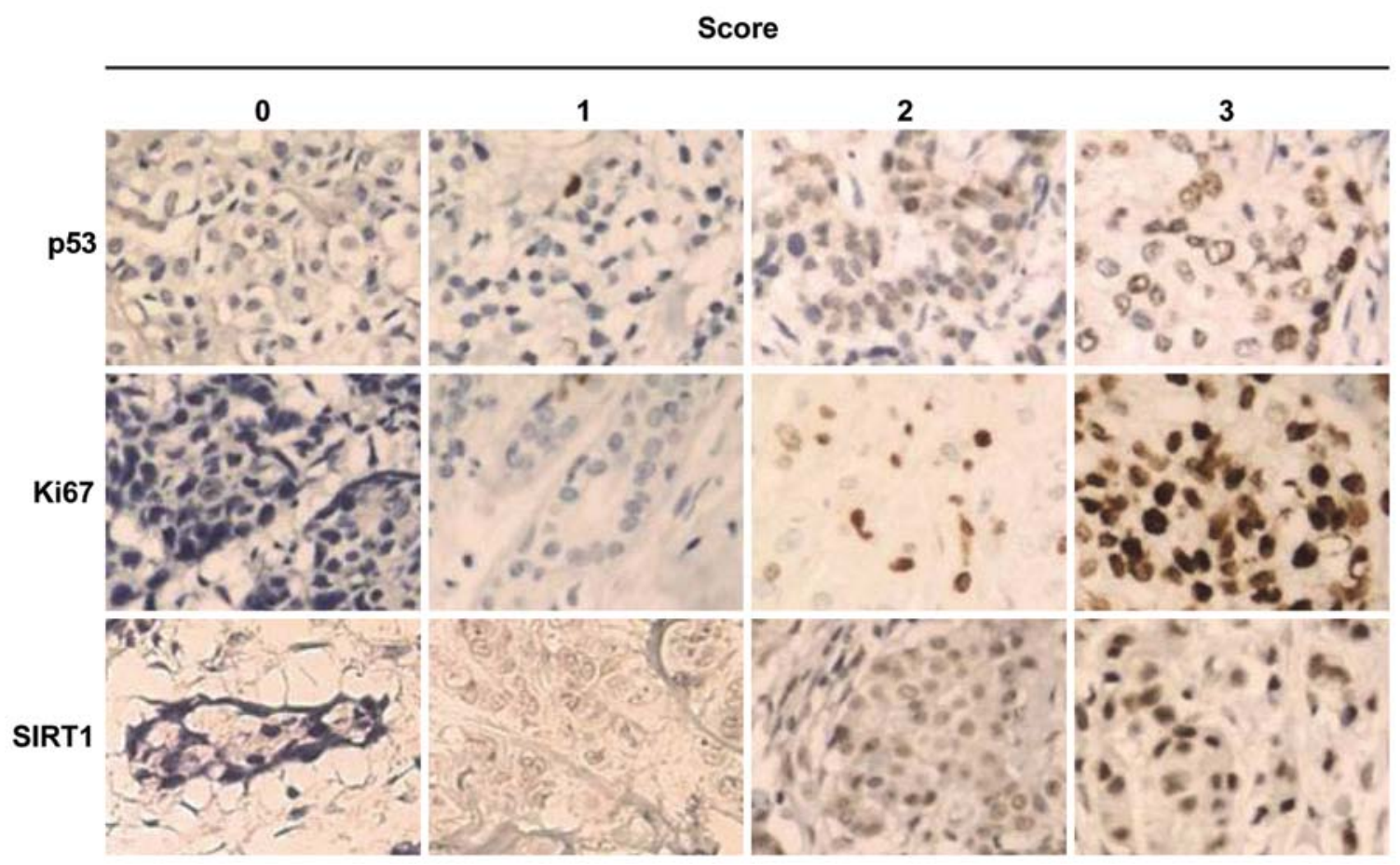

Figure 1. Representative results of immunohistochemical analysis (x200). Top, p53; middle, Ki67; bottom, SIRT1. The staining intensity from negative to high score is 0 to 3 (from left to right panel).

A

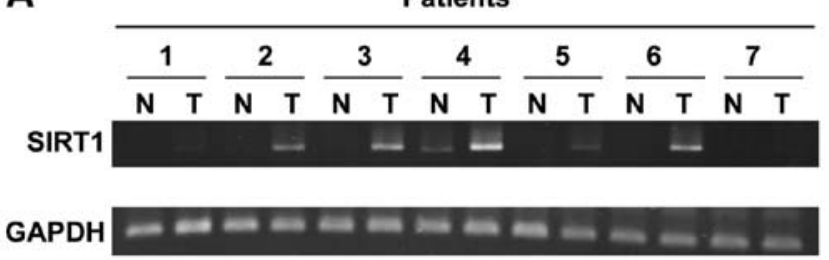

B

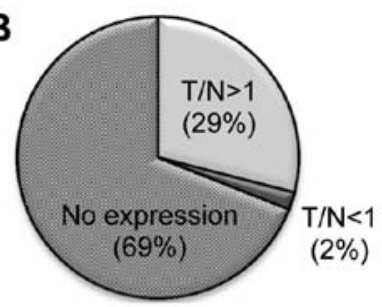

Figure 2. SIRT1 mRNA expression in breast cancer patients. (A) The SIRT1 mRNA expression in representative cases. GAPDH acted as an internal control. (B) The quantitative results. $\mathrm{T}$, tumor tissue; $\mathrm{N}$, paired normal tissue.

was collected and stored at $-20^{\circ} \mathrm{C}$ until analysis. The protein concentration was determined using the bicinchoninic acid protein assay (Pierce, Rockford, IL, USA) according to the manufacturer's instructions. Fifty micrograms of protein were separated by SDS-PAGE and then transferred onto a PVDF membrane (0.4 $\mu \mathrm{m}$; Millipore, Billerica, MA, USA). Non-specific interactions were blocked by incubating the membrane with 5\% non-fat dry milk in $0.5 \%$ PBST for $1 \mathrm{~h}$ at room temperature. The membrane was then blotted with anti-SIRT1, Bcl-2, or $\beta$-actin antibodies at $4^{\circ} \mathrm{C}$ overnight. HRP-conjugated secondary antibodies were added for $1 \mathrm{~h}$, and the immunoreactive signals were then detected using the SuperSignal West Pico Chemiluminescent Substrate (Pierce). The level of protein expression was analyzed using the Gel Image Analysis System (Alpha Innotech Co.).

Statistical analysis. Statistical analyses were performed using the Student's t-test. A P-value of $<0.05$ was considered to indicate a statistically significant difference. All data are shown as the means \pm SEM. Statistical calculations were performed using SPSS software (SPSS, Chicago, IL, USA).

\section{Results}

Increased gene expression of SIRT1 in breast cancer. The results of immunohistochemical analysis revealed that SIRT1, Ki67 and p53 were localized in tumor and in normal cells with varying intensities (Fig. 1). The expression of SIRT1 and Ki67 was significantly higher in tumor tissue than in normal tissue $(\mathrm{P}<0.001$ and $\mathrm{P}<0.005$ for SIRT1 and Ki67, respectively). Twenty-three (82.1\%) tumor cases had a higher level of SIRT1 and Ki67 proteins. Furthermore, the RT-PCR results showed that the mRNA level of SIRT1 was overexpressed in breast tumor tissues (29\%), compared to the paired normal tissues (Fig. 2).

Sirtinol induces cell growth inhibition through cell cycle arrest in the G0/G1 phase in breast cancer cells. When cells were treated with sirtinol at higher concentrations $(30-40 \mu \mathrm{M})$, cell shrinkage was observed (Fig. 3A). The cell viability was reduced by sirtinol in a time- and dose-dependent manner in both MCF-7 and MDA-MB-23 cells (Fig. 3B). Flow cytometry analysis was used to examine the effects of sirtinol on the cell 
A

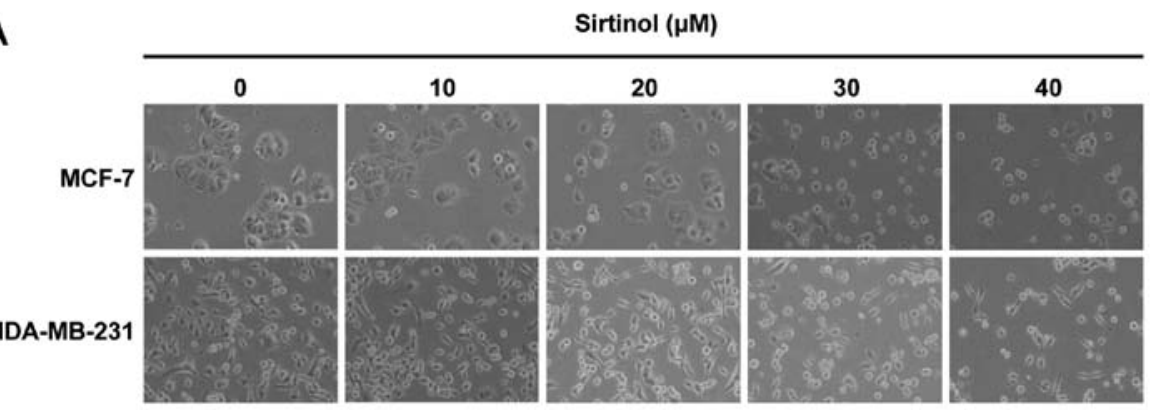

B

MCF-7

MDA-MB-231
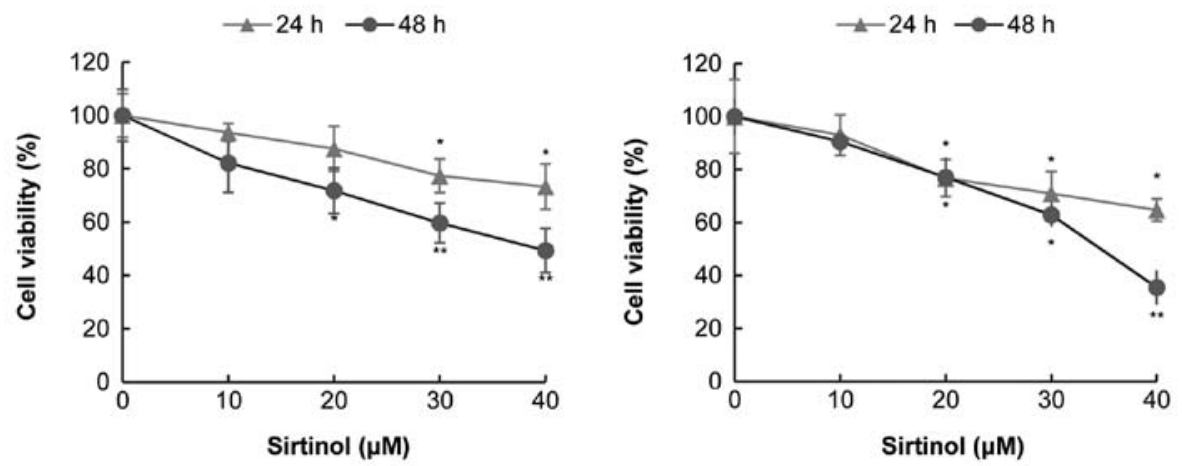

Figure 3. Effects of sirtinol on cell morphology and cellular viability. (A) Cellular morphological changes following sirtinol treatment after $24 \mathrm{~h}$. (B) Cells were treated with the indicated doses of sirtinol for 24 and $48 \mathrm{~h}$, respectively. Prior to each time course, cells were added to a $10 \%$ Alamar blue solution in culture medium and incubated for $6 \mathrm{~h}$. Values were obtained from three independent experiments and are expressed as the means \pm SEM. ${ }^{*} \mathrm{P}<0.05$ and ${ }^{* *} \mathrm{P}<0.01$ between control and sirtinol-treated cells.

A

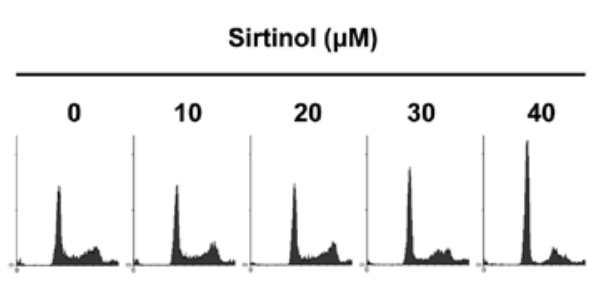

B

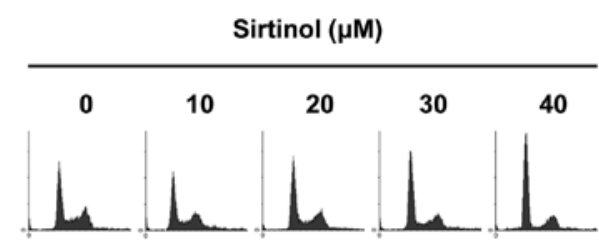

$\square 0 \mu \mathrm{M} \square 10 \mu \mathrm{M} \square 20 \mu \mathrm{M}$ 옹 $30 \mu \mathrm{M} \square 40 \mu \mathrm{M}$

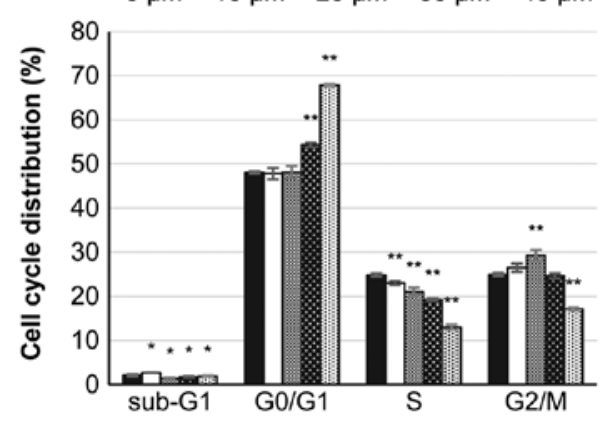

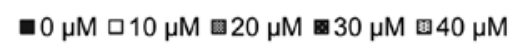

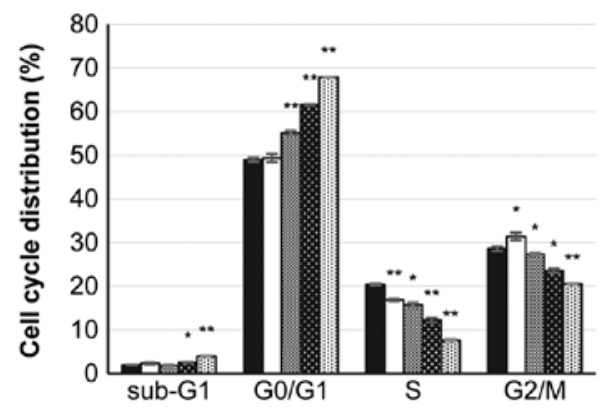

Figure 4. Effect of sirtinol on the cell cycle progression of breast cancer cells. (A) MCF-7 and (B) MDA-MB-231 cells were treated with or without increasing concentrations of sirtinol for $24 \mathrm{~h}$, respectively. Cells were fixed, stained and analyzed for DNA content. Data are presented as the means \pm SD ( $\mathrm{n}=3$ ). ${ }^{*} \mathrm{P}<0.005$ and ${ }^{* *} \mathrm{P}<0.001$ between control and sirtinol-treated cells.

cycle progression of breast cancer cells. As shown in Fig. 4, sirtinol induced a cell cycle arrest in the G0/G1 phase in both cell lines. The proportion of the sub-G1 population also increased slightly in MDA-MB-231 cells, but not in MCF-7 cells.
Sirtinol attenuates the abundance of pro-survival Bcl-2 protein. To characterize the molecular mechanisms underlying these different behaviors, we analyzed the protein expression of $\mathrm{Bcl}-2$ by western blotting. The Bcl-2 protein 
A

MCF-7
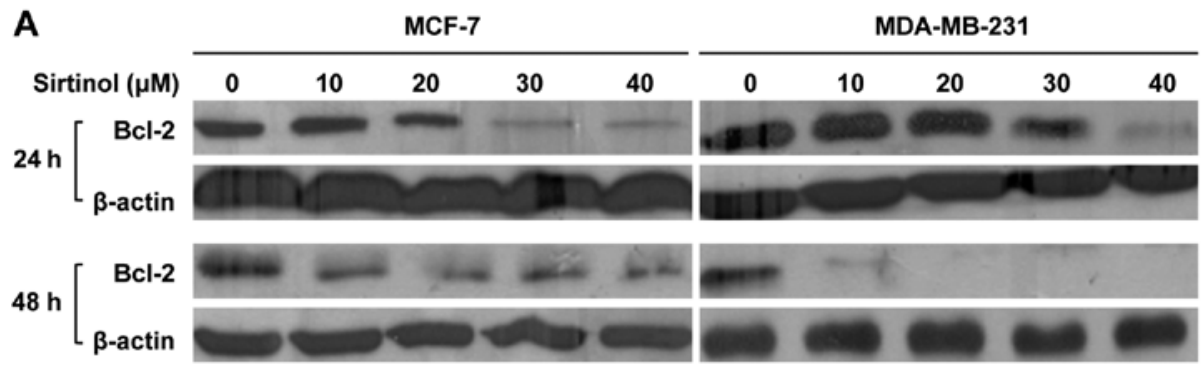

B

MCF-7

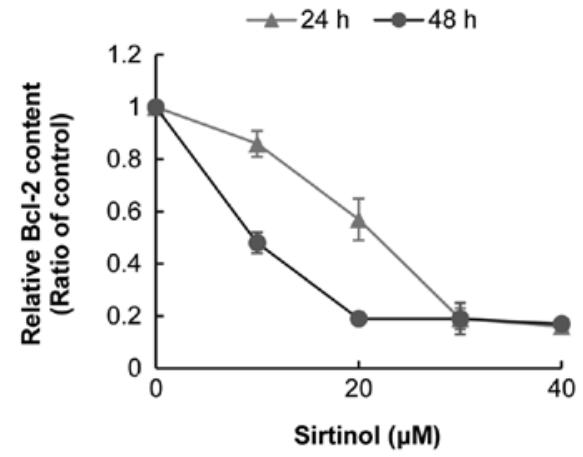

MDA-MB-231

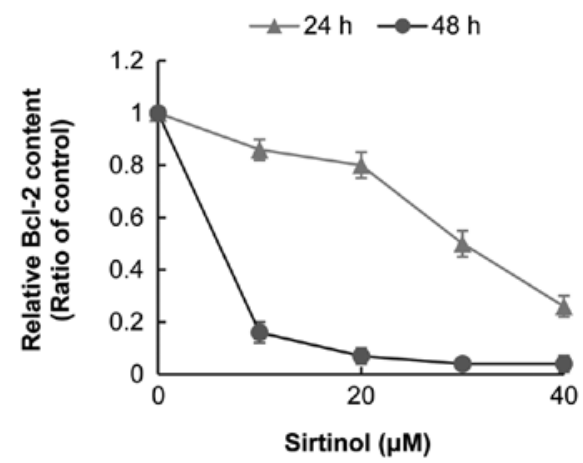

Figure 5. Expression of the Bcl-2 protein in the sirtinol-treated human breast cancer cell lines, (A) MCF-7 and (B) MDA-MB-231. Cells were cultured for the indicated periods with increasing doses of sirtinol $(0-40 \mu \mathrm{M})$ for 24 and $48 \mathrm{~h}$, respectively. $\beta$-actin acted as an internal control.

level in MCF-7 and MDA-MB-231 cells was significantly decreased with sirtinol treatment, particularly with 48 -h treatment (Fig. 5).

\section{Discussion}

The expression of SIRT1 in breast cancer tissues is controversial. Kuzmichev et al (8) and Sung et al (27) demonstrated an overexpression of the SIRT1, while Wang et al (23) reported a decreased expression of the SIRT1 protein in breast tumor tissues. This study showed that there was an extremely high frequency of overexpressed SIRT1 in breast tumor tissues compared to their paired normal tissues $(92.59 \%, \mathrm{n}=27)$ (Fig. 1). Notably, the immuhistochemistry results indicated that there is a positive and high correlation of Ki67 and SIRT1 expression (Fig. 1A). It was also found that breast cancer tissue with less proliferative potential (Ki67 scores 0 and 1) exerts a lower expression level of SIRT1, suggesting that SIRT1 could be a predictive marker for the prognosis of breast cancer. Therefore, we proposed that an overexpression of SIRT1 may be involved in breast tumorigenesis, particularly in breast tumor promotion.

In general, estrogen receptor (ER)-negative breast cancer is often more aggressive and drug-resistant than ER-positive breast cancer. To examine the roles of SIRT1 in breast cancer cells, two human breast cancer cell lines, MCF-7 (ER-positive) and MDA-MB-231 (ER-negative), were examined. We demonstrated that sirtinol effectively induced proliferation inhibition in both MCF-7 and MDA-MB-231 cells, indicating the promising therapeutic strategy of targeting SIRT1 for breast cancer without having to consider the ER status.

Furthermore, inhibition of SIRT1 causes an accumulation of $\mathrm{G} 0 / \mathrm{G} 1$ phase and a decrease in both $\mathrm{S}$ and $\mathrm{G} 2 / \mathrm{M}$ phases
(Fig. 3), suggesting the role of SIRT1 in cell cycle progression. Furthermore, we explored whether sirtinol inhibits cell proliferation through the apoptosis pathway in MCF-7 and MDA-MB-231 cells. Several studies reported that knockdown of SIRT1 or inhibition of SIRT1 activity, such as sirtinol, splitomycin, and cambinol (28-30), causes an apoptotic death of cancer cells.

Among the proteins involved in the control of the apoptotic pathway, Bcl-2, a pro-survival protein, has been demonstrated to protect cells from apoptosis (31). Bcl-2 inhibits the mitochondrial pathways of apoptosis through local effects at the mitochondrial and endoplasmic reticulum membranes (32). Furthermore, Bcl-2 was reported to be overexpressed in most breast cancer cells $(33,34)$. Therefore, downregulation of $\mathrm{Bcl}-2$ is a potential chemotherapeutic strategy (35). Our data showed that sirtinol promoted apoptosis by decreasing the Bcl-2 expression in both MCF-7 and MDA-MB-231 cells.

The results suggest that inhibition of SIRT1 has therapeutic potential as an antitumor strategy. The present study confirmed the correlation between SIRT1 and the prognosis of breast cancer patients. Markedly, the blockade of SIRT activity causes an anti-breast cancer cell effect through downregulating the Bcl-2 protein. Therefore, the specific targeting of the SIRT1 protein may be a beneficial chemotherapeutic strategy, particularly for ER-negative breast cancer.

\section{Acknowledgements}

This study was supported by a grant from and the Changhua Christian Hospital-Kaohsiung Medical University joint grant (97-CCH-KMU-014), Taiwan. Editorial support was provided by Ms. Yu-Fen Wang, M.S. 


\section{References}

1. Blander G and Guarente L: The Sir2 family of protein deacetylases. Annu Rev Biochem 73: 417-435, 2004.

2. Michan S and Sinclair D: Sirtuins in mammals: insights into their biological function. Biochem J 404: 1-13, 2007.

3. Vaziri H, Dessain SK, Ng Eaton E, et al: hSIR2(SIRT1) functions as an NAD-dependent p53 deacetylase. Cell 107: 149-159, 2001.

4. Dai JM, Wang ZY, Sun DC, Lin RX and Wang SQ: SIRT1 interacts with p73 and suppresses p73-dependent transcriptional activity. J Cell Physiol 210: 161-166, 2007.

5. Wong S and Weber JD: Deacetylation of the retinoblastoma tumour suppressor protein by SIRT1. Biochem J 407: 451-460, 2007.

6. Huffman DM, Grizzle WE, Bamman MM, et al: SIRT1 is significantly elevated in mouse and human prostate cancer. Cancer Res 67: 6612-6618, 2007.

7. Jung-Hynes B and Ahmad N: Role of p53 in the anti-proliferative effects of Sirt1 inhibition in prostate cancer cells. Cell Cycle 8: 1478-1483, 2009

8. Kuzmichev A, Margueron R, Vaquero A, et al: Composition and histone substrates of polycomb repressive group complexes change during cellular differentiation. Proc Natl Acad Sci USA 102: 1859-1864, 2005.

9. Zhang Y, Zhang M, Dong H, et al: Deacetylation of cortactin by SIRT1 promotes cell migration. Oncogene 28: 445-460, 2009.

10. Stünkel W, Peh BK, Tan YC, et al: Function of the SIRT1 protein deacetylase in cancer. Biotechnol J 2: 1360-1368, 2007.

11. Kabra N, Li Z, Chen L, et al: SirT1 is an inhibitor of proliferation and tumor formation in colon cancer. J Biol Chem 284 18210-18217, 2009.

12. Liu G, Yuan X, Zeng Z, et al: Analysis of gene expression and chemoresistance of $\mathrm{CD} 133^{+}$cancer stem cells in glioblastoma. Mol Cancer 5: 67, 2006.

13. Jang KY, Hwang SH, Kwon KS, et al: SIRT1 expression is associated with poor prognosis of diffuse large B-cell lymphoma. Am J Surg Pathol 32: 1523-1531, 2008.

14. Bradbury CA, Khanim FL, Hayden R, et al: Histone deacetylases in acute myeloid leukaemia show a distinctive pattern of expression that changes selectively in response to deacetylase inhibitors. Leukemia 19: 1751-1759, 2005.

15. Yuan Z, Zhang X, Sengupta N, Lane WS and Seto E: SIRT1 regulates the function of the Nijmegen breakage syndrome protein. Mol Cell 27: 149-162, 2007.

16. Chu F, Chou PM, Zheng X, Mirkin BL and Rebbaa A: Control of multidrug resistance gene mdrl and cancer resistance to chemotherapy by the longevity gene sirt1. Cancer Res 65: 10183-10187, 2005.

17. Cheng HL, Mostoslavsky R, Saito S, et al: Developmental defects and p53 hyperacetylation in Sir2 homolog (SIRT1)-deficient mice. Proc Natl Acad Sci USA 100: 10794-10799, 2003.
18. Matsushita N, Takami Y,Kimura M, et al: Role of NAD-dependent deacetylases SIRT1 and SIRT2 in radiation and cisplatin-induced cell death in vertebrate cells. Genes Cells 10: 321-332, 2005.

19. Kojima K, Ohhashi R, Fujita Y, et al: A role for SIRT1 in cell growth and chemoresistance in prostate cancer PC3 and DU145 cells. Biochem Biophys Res Commun 373: 423-428, 2008.

20. Yuan J, Minter-Dykhouse K and Lou Z: A c-Myc-SIRT1 feedback loop regulates cell growth and transformation. J Cell Biol 185: 203-211, 2009

21. Firestein R, Blander G, Michan S, et al: The SIRT1 deacetylase suppresses intestinal tumorigenesis and colon cancer growth. PLoS One 3: e2020, 2008.

22. Chua KF, Mostoslavsky R, Lombard DB, et al: Mammalian SIRT1 limits replicative life span in response to chronic genotoxic stress. Cell Metab 2: 67-76, 2005.

23. Wang RH, Sengupta K, Li C, et al: Impaired DNA damage response, genome instability, and tumorigenesis in SIRT1 mutant mice. Cancer Cell 14: 312-323, 2008.

24. Lim CS: SIRT1: tumor promoter or tumor suppressor? Med Hypotheses 67: 341-344, 2006.

25. Li K and Luo J: The role of SIRT1 in tumorigenesis. N Am J Med Sci 4: 104-106, 2011.

26. Tavassoli FA and Devilee P (eds): World Health Organization Classification of Tumours. Pathology and Genetics of Tumours of the Breast and Female Genital Organs. IARC Press, Lyon, 2003.

27. Sung JY, Kim R, Kim JE and Lee J: Balance between SIRT1 and DBC1 expression is lost in breast cancer. Cancer Sci 101: $1738-1744,2010$

28. Heltweg B, Gatbonton T, Schuler AD, et al: Antitumor activity of a small-molecule inhibitor of human silent information regulator 2 enzymes. Cancer Res 66: 4368-4377, 2006.

29. Ota H, Tokunaga E, Chang K, et al: Sirt1 inhibitor, Sirtinol, induces senescence-like growth arrest with attenuated Ras-MAPK signaling in human cancer cells. Oncogene 25: 176-185, 2006.

30. Ford J, Jiang M and Milner J: Cancer-specific functions of SIRT1 enable human epithelial cancer cell growth and survival. Cancer Res 65: 10457-10463, 2005.

31. Reed JC: Bcl-2-family proteins and hematologic malignancies: history and future prospects. Blood 111: 3322-3330, 2008.

32. Hockenbery DM: Targeting mitochondria for cancer therapy. Environ Mol Mutagen 51: 476-489, 2010.

33. Amundson SA, Myers TG, Scudiero D, Kitada S, Reed JC and Fornace AJ Jr: An informatics approach identifying markers of chemosensitivity in human cancer cell lines. Cancer Res 60: 6101-6110, 2000.

34. Zhang M, Guo R, Zhai Y and Yang D: LIGHT sensitizes IFNgamma-mediated apoptosis of MDA-MB-231 breast cancer cells leading to down-regulation of anti-apoptosis Bcl-2 family members. Cancer Lett 195: 201-210, 2003.

35. Kelly PN and Strasser A: The role of Bcl-2 and its pro-survival relatives in tumourigenesis and cancer therapy. Cell Death Differ 18: 1414-1424, 2011. 